\title{
Exposure to heavy metals causes histopathological changes and alters antioxidant enzymes in fresh water fish (Oreochromis niloticus)
}

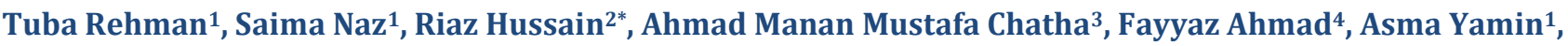 \\ Rabia Akram5, Huma Naz ${ }^{6}$, Ansar Shaheen ${ }^{1}$ \\ ${ }^{1}$ Department of Zoology, Government Sadiq College Women University, Bahawalpur, Pakistan \\ ${ }^{2}$ Department of Pathology, Faculty of Veterinary and Animal Sciences, The Islamia University of Bahawalpur, Pakistan \\ ${ }^{3}$ Department of Entomology, University College of Agriculture and Environmental Sciences, The Islamia University of \\ Bahawalpur, Pakistan; ${ }^{4}$ Institute of Microbiology, University of Agriculture, Faisalabad, Pakistan \\ ${ }^{5}$ Institute of Pure and Applied Biology, Zoology Division, Bhauddin Zakariya University, Multan, Pakistan \\ ${ }^{6}$ Cholistan University of Veterinary and Animal Sciences, Bahawalpur, Pakistan
}

\section{Received:}

March 20, 2020

Accepted:

August 16, 2020

Online First:

October 17, 2020

Published:

January 30, 2021

*Corresponding author email: dr.riaz.hussain@iub.edu.pk

\begin{abstract}
Expansion in technology, development in industries and extensive applications of synthetic chemical have become major regulatory issues toward the public health, wildlife and aquatic ecosystems. Therefore, the monitoring and investigation of environmental contaminants in order to mitigate the adverse impacts is crucial to save public health and aquatic wildlife. The present study describes the deleterious effects of heavy metals at low levels in fresh water fish. A total of 120 fish of same age and body mass were arbitrarily distributed and allocated into eight groups (A-H). Group A was run parallel to other groups and maintained as control group. Collected data was analyzed by using Tukey's Test. Results indicated various clinical ailments including erratic swimming and jerking movement. Microscopic observation of gills, liver and brain tissues showed different histopathological changes in fish exposed to various heavy metals. The antioxidant enzymes activities were increased or decreased in a concentration and enzyme dependent manner. Exposure of treatments showed significant increase in peroxidase and glutathione-s-transferase but decreased catalase and superoxide dismutase activity at higher exposure to heavy metals. Regarding overall toxicity, nickel was least sensitive towards the fish, but tertiary metal mixture was more toxic to fish (Oreochromis niloticus) followed by binary mixture. In conclusion, results of our study suggested that the exposure to different heavy metals even at low concentrations poses serious concern towards their potential danger to the survival and growth of fish and induces deleterious effects in gills, brain and liver tissues.
\end{abstract}

Keywords: Heavy Metals, Oreochromis niloticus, Liver, Antioxidant Enzymes, Histopathology

\section{How to cite this:}

Rehma T, Naz S, Hussain R, Chatha AMM, Ahmad F, Yamin A, Akram R, Naz H and Shaheen A, 2021. Exposure to heavy metals causes histopathological changes and alters antioxidant enzymes in fresh water fish (Oreochromis niloticus). Asian J. Agric. Biol. 2021(1). DOI: https://doi.org/10.35495/ajab.2020.03.143

This is an Open Access article distributed under the terms of the Creative Commons Attribution 3.0 License. (https://creativecommons.org/licenses/by/3.0), which permits unrestricted use, distribution, and reproduction in any medium, provided the original work is properly cited. 


\section{Introduction}

Aquatic organisms are frequently used for toxicity tests, organizing and protecting aquaculture environment (Ghaffar et al., 2019; Ghaffar et al., 2020). Recently, aquatic pollution has increased due to indiscriminate and extensive use of primary nutrients like nitrogen and phosphorus. Different human activities are responsible for the increased rate of pollutants in the aquatic environment in association with industrial chemicals, pesticides and heavy metals. Due to the large concentration of organic pollutants in the aquatic environment, the amount of oxygen reduces and leads to increase rate of mortality and normal functioning of exposed aquatic organisms. Investigation and exact information about the toxic effects related to health of aquatic organisms, survival rate and random variations in physicochemical conditions of an organism is of vital importance (Naz et al., 2020).

Aquatic organisms including fish, plants, invertebrates, and vertebrates are the important assets of nature which overcome the huge stress produced by anthropogenic activities of human beings (Varsha et al., 2017). Studies have shown that the aquatic environment is mostly polluted by the industrial effluents, household activities and agriculture runoff (Ghaffar et al., 2018a; Hussain et al., 2019; Ghaffar et al., 2020) which causes the ecotoxicological effects and major environmental health issues (Bhattacharya, 2016; Ghaffar et al., 2018b). It is recorded that transfer of toxic substances including, drug residues, heavy metal, insecticides, fungicides, herbicides and various other industrial wastes to humans occurs via consumption of contaminated agricultural products, sediments, zooplankton, phytoplankton, aquatic weeds and fishes (Monferrán et al., 2016). Aquatic organisms like fish are very delicate species among the invertebrates and exhibit almost all the physical and biochemical changes due to exposure of toxic chemicals and are frequently used as the warning tool to monitor the normal status of aquatic ecosystem. Fish are the most important and well known species among various organisms in the aquatic food chain (Taweel et al., 2011) due to their ability to concentrate, metabolize and absorption of waterborne harmful materials. Aquatic organisms are very sensitive to the higher exposure of metals due to their ability to produce the highly reactive oxygen species by oxidative stress (Nishida, 2011; Sevcikova et al., 2011; Karra et al., 2015). The mechanism toxicity of metal expressed as the production of reactive radicals of oxygen which interacts with the nuclei, proteins and lipids acids resulting in the genetic, cellular and metabolic diseases which cause the death of the living being (Kurutas et al., 2009; Sunaina and Ansari, 2016). The metabolic, cellular and hereditary responses occur due to the oxidative stresses. The antioxidant can stop the oxidative stress produced due to the harmful materials (Al-Asgah et al., 2015). In fishes, the antioxidant defense mechanisms depend on the low molecular weight and enzyme system antioxidants. Exposure to heavy metals can cause the dose and time-dependent oxidative stresses in the fishes due to the high production of reactive oxygen species, high level of peroxidases and low level of glutathione in the fishes (Maran et al., 2009). Previous studies have indicated different harmful effects of different heavy metals and toxicants like reduced growth, altered immune response, induction of oxidative stress, genotoxic effects, and biochemical variations (Ajani and Akpoilih, 2010; Faheem et al., 2012; Ghaffar et al., 2016; Sattar et al., 2016 and Ghaffar et al., 2019). Therefore, this study was designed to determine the various biomarkers including activities of antioxidant enzymes in various tissues of fresh water fish (Oreochromis niloticus) exposed to different heavy metals.

\section{Material and Methods}

\section{Experimental site}

All the research work of this experiment was conducted at Toxicology Laboratory of Zoology Department, Government Sadiq College Women University, Bahawalpur, Pakistan. The experimental trial was designed to check the deleterious effects of nickel, chromium and lead alone and with their possible potential mixtures on freshwater fish (Oreochromis niloticus).

\section{Laboratory conditions and experimental fish management}

Fish $(\mathrm{n}=120)$ were purchased from local fish breeding center and were acclimatized to laboratory condition for time period of 14 days in a natural photoperiod of 12:12 h light and dark cycle before the start of experiment. For acclimatization purpose, the test specimens were kept in glass aquarium having about 100 L water separately supplemented with oxygenators to provide suitable amount of oxygen. During acclimatization, the fish were fed on 


\section{Tuba Rehman et al.}

commercial pellets (25-30\% protein).Water quality parameters including $\mathrm{pH}$ (7.25), temperature (28$\left.30^{\circ} \mathrm{C}\right)$ and hardness $\left(229 \mathrm{mgL}^{-1}\right)$ were measured prior to start of research trial. Water of the entire glass aquarium was replaced twice every $24 \mathrm{~h}$ with routine cleaning of aquaria to prevent accumulation of wastes and decaying food particles. The body wet weight of each fish and total length was measured after collection. The fish used in this study weighed between 70 to 100 grams.

Table-1. Physico-chemical analysis of water for experimental fish, Oreochromis niloticus

\begin{tabular}{|l|c|}
\hline \multicolumn{1}{|c|}{ Parameters } & Values \\
\hline Water temperature $\left({ }^{\circ} \mathrm{C}\right)$ & $25.9 \pm 0.45$ \\
\hline Electrical conductivity at $25^{\circ}(\mu \mathrm{mhos} / \mathrm{cm})$ & $411.3 \pm 3.15$ \\
\hline Dissolved oxygen $(\mathrm{mg} / \mathrm{L})$ & $7.89 \pm 0.13$ \\
\hline Alkalinity $(\mathrm{mg} / \mathrm{L})$ & $171.5 \pm 1.43$ \\
\hline Total dissolved solid $(\mathrm{mg} / \mathrm{L})$ & $173.9 \pm 4.34$ \\
\hline $\mathrm{Ph}$ & $7.3 \pm 0.07$ \\
\hline Total hardness $(\mathrm{CaCO}, \mathrm{mg} / \mathrm{L})$ & $169.88 \pm 1.73$ \\
\hline Potassium $(\mathrm{mg} / \mathrm{L})$ & $1.61 \pm 0.13$ \\
\hline Sodium $(\mathrm{mg} / \mathrm{L})$ & $11.8 \pm 0.33$ \\
\hline
\end{tabular}

Test chemicals and preparation of stock solutions

The heavy metals used in this experiment were received as metallic salts. These chemicals were of analytical grades. Heavy metals like lead as $\mathrm{PbCl}_{2}$ (molecular weight $278.11 \mathrm{~g} / \mathrm{mol}$ and purity $99.5 \%$ ), Chromium (molecular weight $266.458 \mathrm{~g} / \mathrm{mol}$ and purity 99.5\%) and Nickel (molecular weight $129.60 \mathrm{~g} / \mathrm{mol}$, purity $99.95 \%$ ) were purchased from local market. From all the metal stock solutions were prepared by dissolving the chemicals in deionized water and then diluted to make required concentrations.

\section{Acute toxicity tests (96-hr)}

Acute toxicity tests were determined with three replicates for fish (Oreochromis niloticus) for individual, binary and tertiary mixtures of metals (Table 2).

\section{Experimental groups}

After acclimatization period of 14 days, fish were randomly divided into eight equal groups (A-H). Each group had 15 fish $(n=15)$. Group A served as control group. Fish were exposed to nickel, chromium and lead at various concentrations for a period of 30 days. Five fish from each group were killed at day 30 of exposure to determine the histopathological and antioxidant enzyme activity. Fish of all groups were carefully observed for any physical and behavioral ailments twice daily.

Table-2. Severity of histopathological in liver, gills and brain of fresh water fish (Oreochroimi sniloticus) exposed to various concentrations of heavy metals.

\begin{tabular}{|c|c|c|c|c|c|c|c|c|}
\hline \multirow{2}{*}{ Histopathological Alterations } & \multicolumn{8}{|c|}{ Groups } \\
\hline & $\mathbf{A}($ control $)$ & $\mathbf{B}(\mathrm{Ni})$ & $\mathrm{C}(\mathrm{Cr})$ & $\mathbf{D}(\mathbf{P b})$ & $\mathrm{E}(\mathrm{Ni}+\mathrm{Cr})$ & $\mathrm{F}(\mathrm{Cr}+\mathrm{Pb})$ & G(Pd+Ni) & $\mathrm{H}(\mathrm{Ni}+\mathrm{Cr}+\mathrm{Pb})$ \\
\hline \multicolumn{9}{|l|}{ Gills } \\
\hline Lamellar atrophy & - & + & ++ & ++ & +++ & ++++ & +++ & ++++ \\
\hline Lamellar disorganization & & ++ & ++ & +++ & +++ & +++ & ++++ & ++++ \\
\hline Epithelial necrosis & - & + & ++ & ++ & ++ & ++ & +++ & ++++ \\
\hline Congestion & - & ++ & + & + & +++ & ++ & +++ & ++++ \\
\hline lamellar fusion & & ++ & + & + & +++ & +++ & +++ & +++ \\
\hline \multicolumn{9}{|l|}{ Liver } \\
\hline Karyorrhexis & - & + & ++ & ++ & +++ & ++++ & +++ & ++++ \\
\hline Nuclear hypertrophy & & ++ & ++ & ++ & +++ & +++ & +++ & ++++ \\
\hline Hepatocellular hypertrophy & - & + & ++ & +++ & ++++ & +++ & +++ & ++++ \\
\hline Eosinophilic infiltration & - & ++ & ++ & + & +++ & ++ & ++++ & ++++ \\
\hline Central Vein contraction & & ++ & ++ & + & +++ & +++ & +++ & +++ \\
\hline \multicolumn{9}{|l|}{ Brain } \\
\hline Necrosis of neurons & - & + & + & + & ++ & +++ & ++ & ++++ \\
\hline Intracellular oedema & - & + & ++ & + & ++ & ++ & ++ & +++ \\
\hline Congestion of neural cells & & + & + & + & +++ & +++ & +++ & +++ \\
\hline
\end{tabular}

Absent (-); Mild (+); moderate $(++)$; severe $(+++)$; very severe $(++++)$ 


\section{Enzyme profiling}

For estimation of various antioxidant enzymes, different body tissues of fish like gills, liver, and brain were removed and stored in bottles containing ice-cold water. Briefly, for removal of erythrocytes, all the organs were washed using phosphate buffer solution having $6.5 \mathrm{pH}$. After that all the organs were triturated using $0.2 \mathrm{~mL}$ normal saline and then homogenate was prepared $(1: 4 \mathrm{w} / \mathrm{v})$ by adding cold buffer. The homogenate from each tissue was centrifuged (10000 $\mathrm{rpm})$ at $4^{0} \mathrm{C}$ for 15 minutes. After centrifugation, the supernatants were removed and placed at $-80^{\circ} \mathrm{C}$ for enzyme profiling. By using UV-visible spectrophotometer, different antioxidant enzymes were such as peroxidase (POD), glutathione-s-transferase (GST), catalase (CAT) and superoxide dismutase (SOD) were measured according to previous protocols (Mannervik, 1985; Giannopolitis and Ries, 1977). The quantity of peroxidase was measured as previously described (Civello et al., 1995). The activity of catalase was determined at a wavelength of $240 \mathrm{~nm}$ (Chance and Maehly, 1955).

\section{Histopathological studies}

For histopathological lesions gills, liver and brain tissues were collected from each fish and fixed in $10 \%$ neutral buffered formalin solution (Hussain et al., 2011; Li et al., 2020). After few days of fixation, all the tissues were processed using standard procedures. About 4-5 $\mu \mathrm{m}$ thick pieces from each tissue were cut with semi-automatic microtome, embedded in paraffin, mounted in DPX and stained with hematoxylin and eosin (Hussain et al., 2019).

\section{Statistical analysis}

The data obtained during the experiments were subjected to ANOVA and the group means were compared by using Tukey's test.

\section{Results}

Results on physical and behavioral parameters showed different abnormalities in treated fish including tremors of fins, erratic swimming, jerking movement, lying on one side, loss of coordination, air gulping, and increased surface breathing and swimming in isolation. No adverse signs were observed in fish of control group.

At histopathological levels liver, gills and brain of fish of control group indicated normal histological structures while various microscopic ailments were observed liver, gills and brain tissues of fish exposed to individual metals and in various mixtures (binary and tertiary mixture). The gills exhibited moderate to severe histopathological changes like disorganization of primary and secondary lamellae, fusion of lamellae, necrosis of epithelial cells and disruption of cartilaginous core in due to exposure to nickel alone. The severity of these changes was increased when fish were exposed to tertiary mixture. The mild to moderate histological changes in brain of the fish were vacuolization and necrosis due to individual exposure. Severe pathological changes observed in the brain of fish exposed to binary and tertiary mixtures such as degeneration of nerve cells, atrophy, swelling of the axon, and cellular damage in the interior and posterior regions were observed. The most damage occurred in the fish exposed to tertiary mixture of nickel, chromium and lead in binary mixtures but least damage occurred when exposed to metals alone. Histopathological changes like central vein congestion, degeneration and necrosis of hepatocytes were also observed in liver of different fish (Table 3).

Table-3. Mean $\mathrm{LC}_{50}$ and lethal concentrations of metals and their mixtures for Oreochromis niloticus at three replicates

\begin{tabular}{|l|c|c|c|c|c|c|c|c|}
\hline \multirow{2}{*}{ Metals } & \multicolumn{4}{|c|}{ LC5 concentration } & \multicolumn{4}{c|}{ Lethal concentration } \\
\cline { 2 - 9 } & R1 & R2 & R3 & Mean \pm SD & R1 & R2 & R3 & Mean \pm SD \\
\hline $\mathrm{A}(\mathrm{Ni})$ & 87.11 & 82.59 & 82.39 & $84.03 \pm 2.67$ & 170.52 & 166.81 & 166.78 & $168.1 \pm 2.1$ \\
\hline $\mathrm{B}(\mathrm{Cr})$ & 68.44 & 70.92 & 71.77 & $70.38 \pm 1.73$ & 133.13 & 127.85 & 134.56 & $131.8 \pm 3.5$ \\
\hline $\mathrm{C}(\mathrm{Pb})$ & 58.80 & 63.29 & 63.55 & $61.88 \pm 2.67$ & 126.56 & 123.61 & 111.63 & $120.6 \pm 7.9$ \\
\hline $\mathrm{D}(\mathrm{Ni}+\mathrm{Cr})$ & 63.47 & 60.04 & 60.67 & $61.39 \pm 1.83$ & 134.93 & 137.78 & 134.80 & $135.8 \pm 1.6$ \\
\hline $\mathrm{E}(\mathrm{Cr}+\mathrm{Pb})$ & 54.46 & 58.54 & 61.68 & $58.89 \pm 2.63$ & 111.68 & 115.68 & 112.85 & $113.4 \pm 2.1$ \\
\hline $\mathrm{F}(\mathrm{Pb}+\mathrm{Ni})$ & 65.65 & 71.13 & 66.14 & $67.64 \pm 1.83$ & 131.90 & 129.30 & 134.57 & $131.9 \pm 1.8$ \\
\hline $\mathrm{G}(\mathrm{Ni}+\mathrm{Cr}+\mathrm{Pb})$ & 53.32 & 53.52 & 51.32 & $52.72 \pm 1.22$ & 138.14 & 124.98 & 146.41 & $136.5 \pm 10.8$ \\
\hline
\end{tabular}

$\mathrm{R} 1=1$ st replication; $\mathrm{R} 2=2$ nd replication; $\mathrm{R} 3=3$ rd replication 


\section{Tuba Rehman et al.}

\section{Antioxidant enzyme activities}

The results on different antioxidant enzymes in different tissues including liver, gills and brain of fish exposed to various concentrations of heavy metals are presented in (tables 4-6). The results indicated that the quantity of antioxidant enzymes catalase (CAT) and superoxide dismutase (SOD) significantly decreased in gills, liver and brain tissues obtained from fish exposed to individual and their binary and tertiary mixtures when compared to untreated fish increased. The quantity of peroxidase (POD) and Glutathione-Stransferase (GST) increased significantly in gills, liver and brain tissues of treated fish compared to untreated fish.

Table-4. Changes in the quantity ( $\left.\mathrm{UmL}^{-1}\right)$ of different antioxidant enzymes in liver of fish (Oreochromis niloticus) exposed to heavy metals Changes in the activity levels $\left(\mathrm{UmL}^{-1}\right)$ of enzymes

\begin{tabular}{|c|c|c|c|c|c|}
\hline $\begin{array}{l}\text { Groups/ } \\
\text { Treatment }\end{array}$ & $\begin{array}{c}\text { Exposure } \\
\text { Level }(\mathrm{mg} / \mathrm{L})\end{array}$ & Catalase (CAT) & $\begin{array}{l}\text { Peroxidase } \\
\text { (POD) }\end{array}$ & $\begin{array}{c}\text { Superoxide } \\
\text { dismutase (SOD) }\end{array}$ & \begin{tabular}{|c|} 
Glutathione-S- \\
transferase (GST)
\end{tabular} \\
\hline \multicolumn{6}{|l|}{$5 \%$ of $\mathrm{LC}_{50}$} \\
\hline A (Control) & 0.00 & $50.83 \pm 0.65 b$ & $0.21 \pm 0.06$ & $38.21 \pm 0.69$ & $40.05 \pm 2.09$ \\
\hline $\mathrm{B}(\mathrm{Ni})$ & 4.20 & $31.88 \pm 1.46^{*}$ & $0.26 \pm 0.02 *$ & $25.27 \pm 0.44 *$ & $48.54 \pm 0.93 *$ \\
\hline $\mathrm{C}(\mathrm{Cr})$ & 3.51 & $30.22 \pm 0.50 *$ & $0.26 \pm 0.02 *$ & $31.78 \pm 0.69^{*}$ & $51.38 \pm 0.96^{*}$ \\
\hline $\mathrm{D}(\mathrm{Pb})$ & 3.09 & $41.48 \pm 0.80 *$ & $0.24 \pm 0.01 *$ & $40.85 \pm 0.348$ & $50.37 \pm 0.75 *$ \\
\hline $\mathrm{E}(\mathrm{Ni}+\mathrm{Cr})$ & 3.06 & $41.48 \pm 0.93 * *$ & $0.25 \pm 0.01 *$ & $48.24 \pm 0.32 * *$ & $52.78 \pm 1.04 *$ \\
\hline $\mathrm{F}(\mathrm{Cr}+\mathrm{Pb})$ & 2.94 & $51.36 \pm 0.45^{* *}$ & $0.24 \pm 0.02 *$ & $50.52 \pm 0.60 * *$ & $64.74 \pm 0.53 * *$ \\
\hline $\mathrm{G}(\mathrm{Pb}+\mathrm{Ni})$ & 3.38 & $47.32 \pm 0.88 * *$ & $0.32 \pm 0.01 * *$ & $31.90 \pm 0.59 *$ & $61.51 \pm 0.928 * *$ \\
\hline $\mathrm{H}(\mathrm{Ni}+\mathrm{Cr}+\mathrm{Pb})$ & 2.63 & $51.36 \pm 0.57 * *$ & $0.29 \pm 0.0^{*}$ & $50.22 \pm 0.40^{* *}$ & $67.82 \pm 0.57 * *$ \\
\hline \multicolumn{6}{|l|}{$7 \%$ of $\mathrm{LC}_{50}$} \\
\hline A (Control) & 0.00 & $50.83 \pm 0.65$ & $0.22 \pm 0.06$ & $38.21 \pm 0.69$ & $40.05 \pm 2.09$ \\
\hline $\mathrm{B}(\mathrm{Ni})$ & 5.88 & $26.48 \pm 0.67 *$ & $0.27 \pm 0.01 *$ & $20.65 \pm 0.29 *$ & $48.66 \pm 0.39 *$ \\
\hline $\mathrm{C}(\mathrm{Cr})$ & 4.92 & $24.83 \pm 0.74 *$ & $0.28 \pm 0.02$ & $25.10 \pm 0.29 *$ & $48.24 \pm 0.33 *$ \\
\hline $\mathrm{D}(\mathrm{Pb})$ & 4.33 & $38.76 \pm 1.25 * *$ & $0.28 \pm 0.01 *$ & $28.44 \pm 0.27 *$ & $59.24 \pm 0.57 * *$ \\
\hline $\mathrm{E}(\mathrm{Ni}+\mathrm{Cr})$ & 4.29 & $38.75 \pm 0.85 * *$ & $0.34 \pm 0.01 * *$ & $32.04 \pm 0.45^{*}$ & $67.89 \pm 0.2 * *$ \\
\hline $\mathrm{F}(\mathrm{Cr}+\mathrm{Pb})$ & 4.12 & $38.88 \pm 0.93 * *$ & $0.33 \pm 0.01 * *$ & $33.46 \pm 0.01 *$ & $75.04 \pm 0.42 * *$ \\
\hline $\mathrm{G}(\mathrm{Pb}+\mathrm{Ni})$ & 4.73 & $39.70 \pm 1.37 * *$ & $0.39 \pm 0.02 * *$ & $27.87 \pm 0.74 *$ & $50.12 \pm 0.11 *$ \\
\hline $\mathrm{H}(\mathrm{Ni}+\mathrm{Cr}+\mathrm{Pb})$ & 3.69 & $39.76 \pm 1.16^{* *}$ & $0.41 \pm 0.01 * *$ & $15.31 \pm 0.87 * *$ & $78.17 \pm 0.47 * *$ \\
\hline \multicolumn{6}{|l|}{$9 \%$ of $\mathrm{LC}_{50}$} \\
\hline A (Control) & 0.00 & $50.83 \pm 0.65$ & $0.22 \pm 0.06$ & $38.21 \pm 0.69$ & $40.05 \pm 2.09$ \\
\hline $\mathrm{B}(\mathrm{Ni})$ & 7.56 & $21.28 \pm 1.07 *$ & $0.38 \pm 0.03 *$ & $11.88 \pm 0.43 *$ & $56.51 \pm 0.97 *$ \\
\hline $\mathrm{C}(\mathrm{Cr})$ & 6.33 & $18.03 \pm 0.39 *$ & $0.34 \pm 0.01 *$ & $12.66 \pm 0.69 *$ & $53.70 \pm 1.18 *$ \\
\hline $\mathrm{D}(\mathrm{Pb})$ & 5.56 & $22.08 \pm 2.33 *$ & $0.51 \pm 0.01 * *$ & $17.26 \pm 0.34 * *$ & $70.91 \pm 0.67 * *$ \\
\hline $\mathrm{E}(\mathrm{Ni}+\mathrm{Cr})$ & 5.52 & $22.06 \pm 0.21 *$ & $0.52 \pm 0.02 * *$ & $27.83 \pm 0.76 * *$ & $79.04 \pm 0.70 * *$ \\
\hline $\mathrm{F}(\mathrm{Cr}+\mathrm{Pb})$ & 5.30 & $26.41 \pm 0.68 * *$ & $0.61 \pm 0.01 * *$ & $28.63 \pm 0.48 * *$ & $72.43 \pm 1.12 * *$ \\
\hline $\mathrm{G}(\mathrm{Pb}+\mathrm{Ni})$ & 6.08 & $24.62 \pm 1.22 * *$ & $0.34 \pm 0.02 *$ & $14.70 \pm 0.93 *$ & $61.11 \pm 0.62 * *$ \\
\hline $\mathrm{H}(\mathrm{Ni}+\mathrm{Cr}+\mathrm{Pb})$ & 4.74 & $30.32 \pm 1.37 * *$ & $0.63 \pm 0.01 * *$ & $31.49 \pm 1.20 * *$ & $88.95 \pm 0.18 * *$ \\
\hline
\end{tabular}

Mean values with asterisk $(*)$ and $(* *)$ in each column are statistically significantly different from control and each other 
Tuba Rehman et al.

Table-5. Changes in the quantity $\left(\mathrm{UmL}^{-1}\right)$ of different antioxidant enzymes in gills of fish (Oreochromis niloticus) exposed to heavy metals Changes in the activity levels $\left(\mathrm{UmL}^{-1}\right)$ of enzymes

\begin{tabular}{|c|c|c|c|c|c|}
\hline $\begin{array}{l}\text { Groups/ } \\
\text { Treatment }\end{array}$ & $\begin{array}{c}\text { Exposure } \\
\text { Level }(\mathrm{mg} / \mathrm{L})\end{array}$ & Catalase (CAT) & $\begin{array}{l}\text { Peroxidase } \\
\text { (POD) }\end{array}$ & $\begin{array}{c}\text { Superoxide } \\
\text { dismutase (SOD) }\end{array}$ & $\begin{array}{c}\text { Glutathione-S- } \\
\text { transferase (GST) }\end{array}$ \\
\hline \multicolumn{6}{|l|}{$5 \%$ of $\mathrm{LC}_{50}$} \\
\hline A (Control) & 0.00 & $47.54 \pm 1.12$ & $0.10 \pm 0.01$ & $36.27 \pm 0.65$ & $32.59 \pm 1.03$ \\
\hline $\mathrm{B}(\mathrm{Ni})$ & 4.20 & $30.21 \pm 1.28 *$ & $0.17 \pm 0.02 *$ & $23.14 \pm 0.40 *$ & $37.54 \pm 0.5 * 2$ \\
\hline $\mathrm{C}(\mathrm{Cr})$ & 3.51 & $25.62 \pm 0.75^{*}$ & $0.16 \pm 0.01 *$ & $29.55 \pm 0.28 *$ & $40.76 \pm 0.63 *$ \\
\hline $\mathrm{D}(\mathrm{Pb})$ & 3.09 & $38.40 \pm 0.40 * *$ & $0.21 \pm 0.01 *$ & $38.75 \pm 0.66^{* *}$ & $42.54 \pm 1.02 *$ \\
\hline $\mathrm{E}(\mathrm{Ni}+\mathrm{Cr})$ & 3.06 & $38.40 \pm 1.07 *$ & $0.22 \pm 0.02 * *$ & $45.60 \pm 0.65^{* *}$ & $44.37 \pm 0.96^{*}$ \\
\hline $\mathrm{F}(\mathrm{Cr}+\mathrm{Pb})$ & 2.94 & $47.70 \pm 0.46^{* *}$ & $0 . .24 \pm 0.02 * *$ & $47.58 \pm 0.53 * *$ & $52.53 \pm 0.54 * *$ \\
\hline $\mathrm{G}(\mathrm{Pb}+\mathrm{Ni})$ & 3.38 & $43.63 \pm 1.28 * *$ & $0.27 \pm 0.01 * *$ & $42.20 \mathrm{c} \pm 0.89 * *$ & $38.96 \pm 0.23 *$ \\
\hline $\mathrm{H}(\mathrm{Ni}+\mathrm{Cr}+\mathrm{Pb})$ & 2.63 & $46.33 \pm 0.59 * *$ & $0.31 \pm 0.01 * *$ & $48.31 \mathrm{a} \pm 0.55^{* *}$ & $60.71 \pm 0.45 * *$ \\
\hline \multicolumn{6}{|l|}{$7 \%$ of $\mathrm{LC}_{50}$} \\
\hline A (Control) & 0.00 & $47.54 \pm 1.12$ & $0.18 \pm 0.01$ & $36.27 \pm 0.65$ & $32.59 \pm 1.03$ \\
\hline $\mathrm{B}(\mathrm{Ni})$ & 5.88 & $24.70 \pm 1.01 *$ & $0.26 \pm 0.02 *$ & $18.40 \pm 0.52 *$ & $39.91 \pm 0.54 *$ \\
\hline $\mathrm{C}(\mathrm{Cr})$ & 4.92 & $21.21 \pm 0.98 *$ & $0.20 \pm 0.01 *$ & $21.79 \pm 0.43 *$ & $37.47 \pm 0.44^{*}$ \\
\hline $\mathrm{D}(\mathrm{Pb})$ & 4.33 & $35.48 \pm 0.16^{*}$ & $0.34 \pm 0.02 *$ & $26.85 \pm 0.61 *$ & $50.07 \pm 0.25 * *$ \\
\hline $\mathrm{E}(\mathrm{Ni}+\mathrm{Cr})$ & 4.29 & $35.48 \pm 0.88 *$ & $0.31 \pm 0.01 *$ & $29.90 \pm 0.49 *$ & $52.44 \pm 0.38 * *$ \\
\hline $\mathrm{F}(\mathrm{Cr}+\mathrm{Pb})$ & 4.12 & $35.70 \pm 0.35 *$ & $0.32 \pm 0.01 *$ & $31.77 \pm 0.40 *$ & $63.00 \pm 0.58 * *$ \\
\hline $\mathrm{G}(\mathrm{Pb}+\mathrm{Ni})$ & 4.73 & $36.77 \pm 0.94 *$ & $0.29 \pm 0.02 * *$ & $25.15 \pm 0.70 *$ & $40.44 \pm 0.39 *$ \\
\hline $\mathrm{H}(\mathrm{Ni}+\mathrm{Cr}+\mathrm{Pb})$ & 3.69 & $36.80 \pm 1.05^{*}$ & $0.55 \pm 0.01 * *$ & $34.88 \pm 1.02 *$ & $70.91 \pm 0.29 * *$ \\
\hline \multicolumn{6}{|l|}{$9 \%$ of $\mathrm{LC}_{50}$} \\
\hline A (Control) & 0.00 & $47.54 \pm 1.12$ & $0.17 \pm 0.01$ & $36.27 \pm 0.65$ & $32.59 \pm 1.03$ \\
\hline $\mathrm{B}(\mathrm{Ni})$ & 7.56 & $17.70 \pm 0.75^{*}$ & $0.30 \pm 0.01 *$ & $10.99 \pm 0.23 *$ & $49.53 \pm 0.73 *$ \\
\hline $\mathrm{C}(\mathrm{Cr})$ & 6.33 & $14.78 \pm 0.85^{*}$ & $0.31 \pm 0.01 *$ & $12.06 \pm 0.66^{*}$ & $40.17 \pm 0.89 *$ \\
\hline $\mathrm{D}(\mathrm{Pb})$ & 5.56 & $22.86 \pm 0.45 *$ & $0.45 \pm 0.01 * *$ & $14.67 \pm 0.61 *$ & $60.47 \pm 0.69 * *$ \\
\hline $\mathrm{E}(\mathrm{Ni}+\mathrm{Cr})$ & 5.52 & $22.85 \pm 0.64^{*}$ & $0.43 \pm 0.02 * *$ & $24.79 \pm 1.27 * *$ & $59.92 \pm 5.18$ \\
\hline $\mathrm{F}(\mathrm{Cr}+\mathrm{Pb})$ & 5.30 & $29.13 \pm 0.74 * *$ & $0.53 \pm 0.02 * *$ & $27.84 \pm 0.53 * *$ & $72.43 \pm 0.56^{* *}$ \\
\hline $\mathrm{G}(\mathrm{Pb}+\mathrm{Ni})$ & 6.08 & $23.18 \pm 0.96^{*}$ & $0.26 \pm 0.01 * *$ & $12.54 \pm 0.73 *$ & $56.54 \pm 1.25^{* *}$ \\
\hline $\mathrm{H}(\mathrm{Ni}+\mathrm{Cr}+\mathrm{Pb})$ & 4.74 & $23.27 \pm 1.00 *$ & $0.11 \pm 0.02 * *$ & $12.45 \pm 0.73^{*}$ & $56.54 \pm 1.25 * *$ \\
\hline
\end{tabular}

Mean values with asterisk $(*)$ and $(* *)$ in each column are statistically significantly different from control and each other 
Tuba Rehman et al.

Table-6. Changes in the quantity $\left(\mathrm{UmL}^{-1}\right)$ of different antioxidant enzymes in brain of fish (Oreochromis niloticus) exposed to heavy metals

\begin{tabular}{|c|c|c|c|c|c|}
\hline $\begin{array}{l}\text { Groups/ } \\
\text { Treatment }\end{array}$ & $\begin{array}{c}\text { Exposure } \\
\text { Level (mg/L) }\end{array}$ & Catalase (CAT) & $\begin{array}{l}\text { Peroxidase } \\
\text { (POD) }\end{array}$ & $\begin{array}{c}\text { Superoxide } \\
\text { dismutase (SOD) }\end{array}$ & $\begin{array}{c}\text { Glutathione-S- } \\
\text { transferase (GST) }\end{array}$ \\
\hline \multicolumn{6}{|c|}{$5 \%$ of $\mathrm{LC}_{50}$} \\
\hline A (Control) & 0.00 & $55.14 \pm 0.74$ & $0.06 \pm 0.01$ & $31.15 \pm 0.15$ & $28.95 \pm 1.03$ \\
\hline $\mathrm{B}(\mathrm{Ni})$ & 4.20 & $36.09 \pm 0.44 *$ & $0.16 \pm 0.01 *$ & $20.25 \pm 0.35^{*}$ & $33.02 \pm 0.98 *$ \\
\hline $\mathrm{C}(\mathrm{Cr})$ & 3.51 & $30.59 \pm 0.57 *$ & $0.17 \pm 0.0 * 1$ & $25.10 \pm 0.77 *$ & $38.99 \pm 0.79 *$ \\
\hline $\mathrm{D}(\mathrm{Pb})$ & 3.09 & $42.03 \pm 0.34 *$ & $0.16 \pm 0.01 *$ & $36.18 \pm 0.46^{*}$ & $43.49 \pm 0.59 * *$ \\
\hline $\mathrm{E}(\mathrm{Ni}+\mathrm{Cr})$ & 3.06 & $42.03 \pm 0.59 *$ & $0.18 \pm 0.02 *$ & $43.95 \pm 0.73 * *$ & $45.48 \pm 1.04 * *$ \\
\hline $\mathrm{F}(\mathrm{Cr}+\mathrm{Pb})$ & 2.94 & $45.20 \pm 0.88$ & $0.26 \pm 0.01 * *$ & $43.74 \pm 0.57 * *$ & $49.74 \pm 1.25^{* *}$ \\
\hline $\mathrm{G}(\mathrm{Pb}+\mathrm{Ni})$ & 3.38 & $46.40 \pm 0.55$ & $0.30 \pm 0.01 * *$ & $40.86 \pm 0.61 * *$ & $39.57 \pm 0.60 * *$ \\
\hline $\mathrm{H}(\mathrm{Ni}+\mathrm{Cr}+\mathrm{Pb})$ & 2.63 & $43.40 \pm 1.05$ & $0.28 \pm 0.01 * *$ & $45.00 \pm 1.25^{* *}$ & $53.70 \pm 0.57 * *$ \\
\hline \multicolumn{6}{|c|}{$7 \%$ of $\mathrm{LC}_{50}$} \\
\hline A (Control) & 0.00 & $55.14 \pm 0.74$ & $0.16 \pm 0.01$ & $31.15 \pm 0.15$ & $28.95 \pm 1.03$ \\
\hline $\mathrm{B}(\mathrm{Ni})$ & 5.88 & $26.78 \pm 0.48 *$ & $0.25 \pm 0.02 *$ & $15.56 \pm 0.37 *$ & $39.26 \pm 0.90 *$ \\
\hline $\mathrm{C}(\mathrm{Cr})$ & 4.92 & $28.07 \pm 0.86^{*}$ & $0.29 \pm 0.01 *$ & $23.13 \pm 0.56^{*}$ & $38.24 \pm 0.93^{*}$ \\
\hline $\mathrm{D}(\mathrm{Pb})$ & 4.33 & $37.18 \pm 0.6^{* *}$ & $0.26 \pm 0.01 *$ & $25.25 \pm 0.43^{*}$ & $43.13 \pm 0.83 *$ \\
\hline $\mathrm{E}(\mathrm{Ni}+\mathrm{Cr})$ & 4.29 & $37.18 \pm 0.76 * *$ & $0.28 \pm 0.01 * *$ & $25.58 \pm 0.35^{*}$ & $55.14 \pm 0.35^{* *}$ \\
\hline $\mathrm{F}(\mathrm{Cr}+\mathrm{Pb})$ & 4.12 & $39.72 \pm 0.90 * *$ & $0.31 \pm 0.02 * *$ & $28.36 \pm 0.39 *$ & $83.37 \pm 0.73 * *$ \\
\hline $\mathrm{G}(\mathrm{Pb}+\mathrm{Ni})$ & 4.73 & $38.07 \pm 0.51 * *$ & $0.33 \pm 0.0 * *$ & $26.22 \pm 0.70 * *$ & $40.44 \pm 0.39 * *$ \\
\hline $\mathrm{H}(\mathrm{Ni}+\mathrm{Cr}+\mathrm{Pb})$ & 3.69 & $42.32 \pm 0.33 * *$ & $0.32 \pm 0.01 * *$ & $33.22 \pm 1.01 * *$ & $64.55 \pm 0.23 * *$ \\
\hline \multicolumn{6}{|c|}{$9 \%$ of $\mathrm{LC}_{50}$} \\
\hline A (Control) & 0.00 & $55.14 \pm 0.74$ & $0.15 \pm 0.01$ & $31.15 \pm 0.15$ & $28.95 \pm 1.03$ \\
\hline $\mathrm{B}(\mathrm{Ni})$ & 7.56 & $18.81 \pm 0.41 *$ & $0.27 \pm 0.02 *$ & $7.91 \pm 0.23^{*}$ & $36.77 \pm 1.12 *$ \\
\hline $\mathrm{C}(\mathrm{Cr})$ & 6.33 & $20.21 \pm 0.72 *$ & $0.25 \pm 0.01 *$ & $10.63 \pm 0.71 *$ & $33.65 \pm 0.82 *$ \\
\hline $\mathrm{D}(\mathrm{Pb})$ & 5.56 & $26.17 \pm 0.48 * *$ & $0.35 \pm 0.02 * *$ & $14.44 \pm 0.87 * *$ & $51.22 \pm 1.06 * *$ \\
\hline $\mathrm{E}(\mathrm{Ni}+\mathrm{Cr})$ & 5.52 & $26.17 \pm 0.63 * *$ & $0.34 \pm 0.03 * *$ & $19.85 \pm 0.23 * *$ & $61.14 \pm 0.85^{* *}$ \\
\hline $\mathrm{F}(\mathrm{Cr}+\mathrm{Pb})$ & 5.30 & $28.42 \pm 0.78 * *$ & $0.50 \pm 0.02 * *$ & $24.52 \pm 0.98 * *$ & $69.32 \pm 1.08 * *$ \\
\hline $\mathrm{G}(\mathrm{Pb}+\mathrm{Ni})$ & 6.08 & $25.84 \pm 1.00 * *$ & $0.44 \pm 0.02 * *$ & $24.14 \pm 0.57 * *$ & $48.55 \pm 0.92 * *$ \\
\hline $\mathrm{H}(\mathrm{Ni}+\mathrm{Cr}+\mathrm{Pb})$ & 4.74 & $32.54 \pm 1.38 * *$ & $0.53 \pm 0.01 * *$ & $26.14 \pm 0.60 * *$ & $76.19 \pm 0.95^{* *}$ \\
\hline
\end{tabular}

Mean values with asterisk (*) and (**) in each column are statistically significantly different from control and each other 


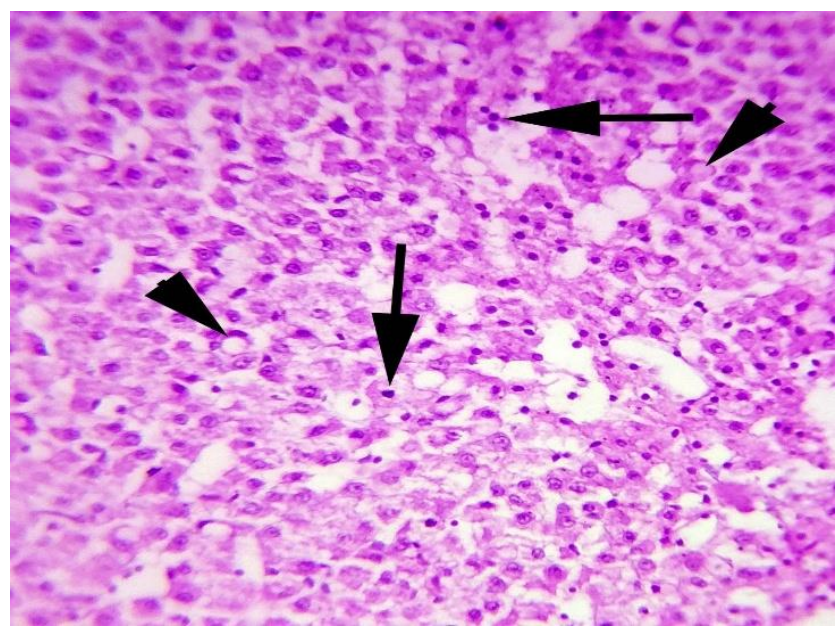

Figure-1. Photomicrograph of liver of fish exposed to heavy metal showing necrosis of hepatocytes (arrows) and vacuolar degeneration (arrow heads). H\&E Stain; 400X

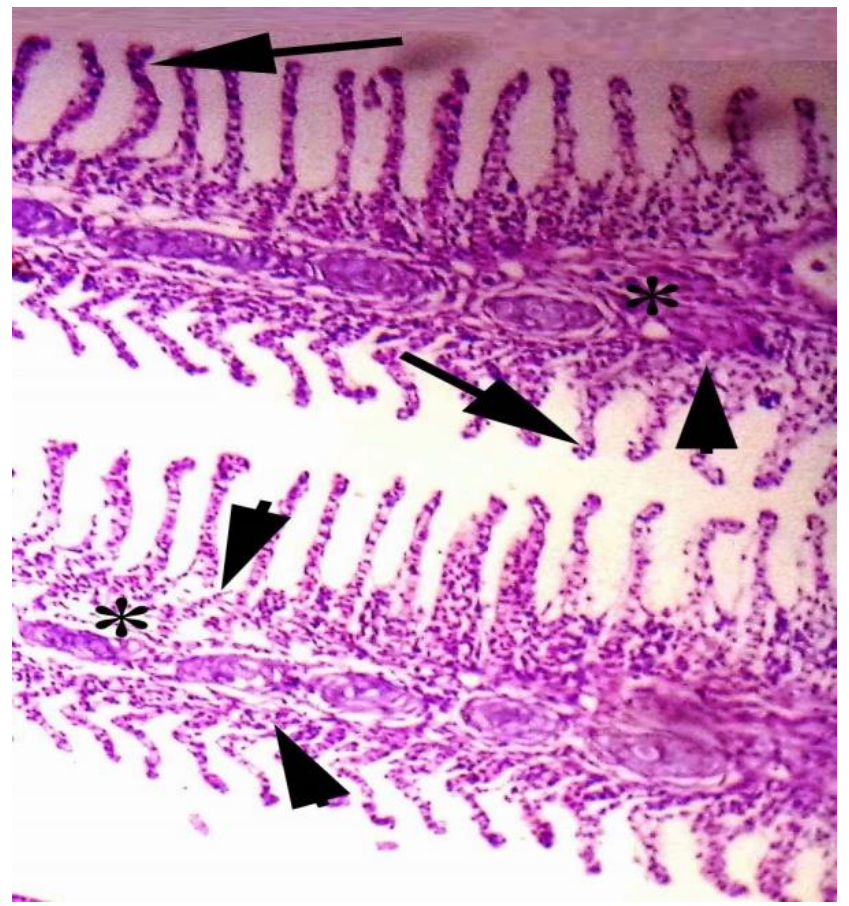

Figure-2. Photomicrograph of gills of fish exposed to heavy metals showing necrosis of epithelial cells of secondary lamellae (arrows), and degeneration and disruption of cartilaginous core (*) and disruption of primary lamellae (arrow heads). H\&E Stain; 400X

\section{Discussion}

The aquatic ecosystem is routinely and extensively polluted due to persistent release of industrial wastes, extensive use of insecticides and pesticides in agriculture, veterinary practice and in public health management. Therefore, monitoring and investigation of deleterious impacts of various pollutants is of vital importance to mitigate their toxic effects in exposed organisms including human beings (Pyle and Wood, 2008; Sattar et al., 2016; Hussain et al., 2018 and Hussain et al., 2019). For measuring the adverse effects of various aquatic pollutants, fishes are considered as a good bio indicator and reflect all the abnormal signs even at very low level of toxicants in aquatic ecosystems (Ding et al., 2000; Ghazanfar et al., 2018 and Ghaffar et al., 2020). Studies have reported that the mechanisms toxicity of various heavy metals are still under debate and depends upon the exposure period, concentrations of metal, concentration of toxicants and their relative affinity, dissolved metals amount and their ratio (Balistrieri and Mebane, 2014). Moreover, it is reported that the bioaccumulation models are very helpful in overcoming the large exposures of heavy metals (Bray and Bettger, 1990). In present study different physical ailments observed in fish might be due to toxic effects of heavy metals including, oxidative damage, neurotoxicity and inflammatory responses (Gaetke and Chow, 2003; Javed et al., 2016; Ghaffar et al., 2016; Sun et al., 2018). Histopathological changesobserved in liver, gills and brain tissues in our study could be due to rapid and increased generation of oxidative stress. Different histopathological changes in rats and broilers have also been observed due to heavy metals (Shahzad et al., 2012; Ghaffar et al., 2017). Previously it has been determined that due to generation of free radicals including reactive oxygen species, the status of is disrupted resulting in induction of structural and fuctions changes in exposed organisms (Poprac et al., 2017). The quantity of antioxidant enzymes (SOD and CAT) were significantly decreased in treated fish. Previously, it is reported that heavy metals like cadmium and copper reduced the performance of CAT in different tissues including heart, gills, and brains of African catfish (Fatima and Usmani, 2013). Moreover, due to chromium exposure, the lower performance of CAT in kidneys and gills of fish (Clarias batrachus) has also been reported (Arshad et al., 2018). The lower values of catalase in our study might be due to oxidative stress (Tripathi and Shasmal, 2011). Furthermore lower concentrations of CAT in various tissues of exposed fish could also be due to accumulation of $\mathrm{H}_{2} \mathrm{O}_{2}$ in the cells leading to poor performance of the cell (Tripathi 
and Yadav, 2015). In present study concentrations of POD and GSH were significantly increased in heavy metal treated fish. Similar reports also available in fish due to heavy metal (Rajeshkumar et al., 2013). Previously it is observed that the activity of peroxidase enzyme increased in brain, liver, muscles, and gills tissues due to iron-treated fish. Due to the 30-day time exposure, significantly higher concentrations of glutathione-S-transferase enzyme have been recorded in brain, gills, and liver tissue due to heavy metals. The increased activity of hepatic GST enzyme due to the cadmium in fish (Oreochromis niloticus) has also been observed (Batool et al., 2018). The significant lower concentrations of CAT and SOD due to binary and tertiary mixtures in gills, liver and brains of fish as compared to individual heavy metal can be related to interactions of metal ions leading to overproduction of free radicals and poor detoxification mechanisms $(\mathrm{Lu}$ et al., 2013). Previously, due to stress induced by toxicants, the poor defensive mechanism in fishes has been reported (Gultekin et al., 2000). As the liver tissue in animals is considered as the main organ of the body which helps in removal of toxins, excretion of wastes and metabolism of toxic substances is sensitive to exposure of toxicants (Sharbidre et al., 2011). Similar to our results regarding poor values of various antioxidants in different tissues of fish due to heavy metals have also been determined (Fatima and Usmani, 2013).

\section{Conclusion}

From the results of our experimental study, it can be augmented that even at very low concentration of individual heavy metals and their mixtures (binary and tertiary) disrupt the histo-architectures, alter the status of different antioxidant enzymes in gills, brain and liver tissues in fish.

\section{Disclaimer: None. \\ Conflict of Interest: None. Source of Funding: None.}

\section{References}

Ajani E and Akpoilih B, 2010. Effect of chronic dietary copper exposure on haematology and histology of common carp (Cyprinus carpio ). J. Appl. Sci. Environ. Manage. 14:222-227.

Al-Asgah NA, Abdel-Warith AEO, Younis ES and Allam HY, 2015. Haematological and biochemical parameters and tissue accumulations of cadmium in Oreochromis niloticus exposed to various concentrations of cadmium chloride. Saudi J. Biol. Sci. 22:543-550.

Ambreen F, Javed M and Batool U, 2015. Tissue specific heavy metals uptake in economically important fish, Cyprinus carpio at acute exposure of metals mixtures. Pak. J. Zool. 47(2): 399-407.

Arshad R, Abdullah S, Naz H and Abbas K, 2018. Catalase Activity as a Bio-Indicator of Lead+ Nickel Toxicity in Carnivorous Fish, Channa striata. Proc. Pak. Acad. Sci.: B. Life Environ. Sci. 55: 37-43.

Balistrieri LS and Mebane CA, 2014. Predicting the toxicity of metal mixtures. Sci. Total Environ. 466: 788-799.

Batool Y, Abdullah, Naz H and Abbas K, 2018. SubLethal Effect of Waterborne Cadmium Exposure on Glutathione S-Transferase and Total Protein Contents in Liver of Carnivorous Fish, Wallago $a t t u$. B Life Environ. Sci. 55: 21-25.

Bhattacharya S, 2016. Changing dimensions and interactions of water crisis and human rights in developing countries. World Sci. News. 34:86-97.

Bray TM and Bettger WJ, 1990. The physiological role of zinc as an antioxidant. Free Radical Biol. Med. 8:281-291. doi: 10.1016/08915849(90)90076-u.

Chance B and Maehly AC, 1955. [136] Assay of catalases and peroxidases. http://dx.doi.org/10.1016/S0076-6879(55)023008

Civello PM, Martinez GA, Chaves AR and Anon MC, 1995. Peroxidase from strawberry fruit (Fragaria ananassa Duch.): partial purification and determination of some properties. J. Agric. Food Chem. 43:2596-2601.

Ding WX, Shen HM and Ong CN, 2000. Microcystic cyanobacteria extract induces cytoskeletal disruption and intracellular glutathione alteration in hepatocytes. Environ. Health Perspec. 108: 605-609.

El Fattah A, 2016. Effect of chromium supplementation on insulin resistance and weight reduction in polycystic ovarian syndrome cases. Sikkim Manipal Univ. Med. J. 3:324-334.

Faheem M, Sulehria AQK, Tariq MI, Khadija A and Fiaz Saeed M, 2012. Effect of sublethal dose of cadmium chloride on biochemical profile and catalase activity in freshwater fish Oreoochromi sniloticus. Biologia. 58: 73-78. 
Fatima M and Usmani N, 2013. Histopathology and bioaccumulation of heavy metals $(\mathrm{Cr}, \mathrm{Ni}$ and $\mathrm{Pb}$ ) in fish (Channa striatus and Heteropneustes fossilis) tissue: A study for toxicity and ecological impacts. Pak. J. Biol. Sci. 16:412-420.

Gaetke LM and Chow CK, 2003. Copper toxicity, oxidative stress, and antioxidant nutrients. Toxicol. 189, 147-163.

Ghaffar A, Hussain R, Abbas G, Ahmad MN, Abbas A, Rahim Y, Younus M, Shahid M and Mohiuddin M, 2018a. Sodium arsenate and/or urea differently affect clinical attributes, hemato-biochemistry and DNA damage in intoxicated commercial layer birds. Toxin Rev. 37: 206-215

Ghaffar A, Hussain R, Aslam M, Abbas G and Khan A, 2016. Arsenic and urea in combination alters the hematology, biochemistry and protoplasm in exposed rohufish (Labeorohita) (Hamilton, 1822). Turkish J. Fish. Aquat. Sci. 16:289-296.

Ghaffar A, Hussain R, Abbas G, Ali MH, Saleem M, Khan T, Malik R and Ahmed H, 2017. Cumulative effects of sodium arsenate and diammonium phosphate on growth performance, hematobiochemistry and protoplasm in commercial layer. Pak. Vet J. 37: 257-262.

Ghaffar A, Hussain R, Abbas G, Kalim M, Khan A, Ferrando S, Gallus L and Ahmed Z, 2018b. Fipronil (Phenylpyrazole) induces hematobiochemical, histological and genetic damage at low doses in common carp, Cyprinus carpio (Linnaeus, 1758). Ecotoxicol. 27:1261-1271

Ghaffar A, Hussain R, Abbas G, Khan R, Akram K, Latif H, Ali S, Baig S, Du X and Khan A, 2019. Assessment of genotoxic and pathologic potentials of fipronil insecticide in Labeo rohita (Hamilton, 1822). Toxin Rev. DOI: 10.1080/15569543.2019.1684321

Ghaffar A, Hussain R, Noreen S, Chodhary IR, Abbas G, Khan A, Ahmed Z, Khan MK, Akram K, Ulhaq M, Ahmad N, Ali F and Niaz M, 2020. Dose and time-related pathological and genotoxic studies on thiamethaxam in fresh water fish (Labeorohita) in Pakistan. Pak. Vet. J. DOI: http://dx.doi.org/10.29261/pakvetj/2020.002

Ghazanfar M, Shahid S and Qureshi IZ, 2018. Vitamin $\mathrm{C}$ attenuates biochemical and genotoxic damage in common carp (Cyprinus carpio) upon joint exposure to combined toxic doses of fipronil and buprofezin insecticides. Aquat. Toxicol. 196: 43-52

Giannopolitis G and Ries S, 1977. In Vitro Production of Superoxide Radical from Paraquat and Its
Interactions by Monuron and Diuron. Weed Sci. 25:298-303.

Gultekin F, Ozturk M and Akdogan M, 2000. The effect of organophosphate insecticide chlorpyrifos-ethyl on lipid peroxidation and antioxidant enzymes (in vitro). Archives of Toxicol. 74:533-538.

Hussain R, Ali F, Rafique A, Ghaffar A and Jabeen G, 2019. Exposure to sub-acute concentrations of glyphosate induce clinico-hematological, serum biochemical and genotoxic damage in adult cockerels. Pak. Vet. J. 39: 181-186.

Hussain R, Ghaffar Al, Ali HM, Abbas RZ, Khan JA, Khan IA, Ahmad I and Iqbal Z, 2018. Analysis of different toxic impacts of Fipronil on growth, hemato-biochemistry, protoplasm and reproduction in adult cockerels. Toxin Rev. 37: 294-303.

Hussain R, Mahmood F, Khan MZ, Khan A and Muhammad F, 2011. Pathological and genotoxic effects of atrazine in male Japanese quail (Coturnix japonica). Ecotoxicol. 20:1-8.

Hussain R, Ghaffar A, Ali HM, Abbas RZ, Khan JA, Khan IA, Ahmad I and Iqbal Z, 2018 Analysis of different toxic impacts of Fipronil on growth, hemato-biochemistry, protoplasm and reproduction in adult cockerels. Toxin Rev. 37: 294-303.

Javed M, Ahmad I, Usmani N and Ahmad M, 2016. Bioaccumulation, oxidative stress and genotoxicity in fish (Channa punctatus) exposed to a thermal power plant effluent. Ecotoxicol. Environ. Saf. 127: 163-169.

Karra S, Satish P, Sunita K and Jyothi NB, 2015. Studies on the biochemical responses in the tissues of freshwater fish Labeo rohita exposed to the organophosphate, phenthoate. Int. J. Pharm. Sci.32:108-115.

Kurutas EB, Şahan A and Altun T, 2009. Oxidative stress biomarkers in liver and gill tissues of spotted barb (Capoeta Barroisi Lortet, 1894) living in Ceyhan river, Adana-Turkey. Turkish J. Biol. 33: 275-282.

Li K, Luo H, Mehmood K, Shahzad M and Li J, 2020. Exploring the potential parasitic pathogens causing diarrheal death to yak calves with bloody excrement through high-throughput sequencing. Agrobiological Records, 1: 1-5. https://doi.org/10.47278/journal.abr/2020.002

Lu Y, Zhang A, Li C, Zhang P, Su X, and Li Y, 2013. The link between selenium binding protein from 
Sinonovacula constricta and environmental pollutions exposure. Fish Shellfish Immunol. 35: 271-277.

Mannervik B, 1985. The isoenzymes of glutathione transferase. Adv. Enzymol. Relat. Areas Mol. Biol. 57:357-417.

Maran E, Fernández M, Barbieri P, Font G and Ruiz MJ, 2009. Effects of four carbamate compounds on antioxidant parameters. Ecotoxicol. Environ. Saf. 72: 922-930.

Monferrán MV, Garnero P, Bistoni MDLA, Anbar AA, Gordon GW and Wunderlin DA, 2016. From water to edible fish. Transfer of metals and metalloids in the San Roque Reservoir (Córdoba, Argentina). Implications associated with fish consumption. Ecol. Indicators. 63: 48-60.

Nishida Y, 2011. The chemical process of oxidative stress by copper (II) and iron (III) ions in several neurodegenerative disorders. Monatshefte fur Chemie-Chemical Monthly. 142:375-384.

Poprac P, Jomova K, Simunkova M, Kollar V, Rhodes CJ and Valko M, 2017. Targeting Free Radicals in Oxidative Stress-Related Human Diseases. Trends Pharmacol. Sci. 38: 592-607.

Pyle G and Wood C, 2008. Radiotracer studies on waterborne copper uptake, distribution, and toxicity in rainbow trout and yellow perch. A comparative analysis. Human Eco. Risk Assess. 14: 243-265.

Rajeshkumar S, Jayaprakas M and Natesan M, 2013. Impact of heavy metals on antioxidant activity in different tissue of milk fish Chanos chanos. Int. J. Appl. Pharm. Technol. 4: 272-279.

Sattar A, Khan A, Hussain HI, He C, Hussain R, Zhiqiang S, Saleemi MK and Gul ST, 2016. Immunosuppressive effects of arsenic in broiler chicks exposed to Newcastle disease virus, J. Immunotoxicol. 13:6, 861-869

Sevcikova MH, Modra H, Slaninova A and Svobodova Z, 2011. Metals as a cause of oxidative stress in fish: a review. Vet Med. 56:537-546.

Sharbidre AA, Metkari V and Patode P, 2011. Effect of methyl parathion and chlorpyrifos on certain biomarkers in various tissues of guppy fish, Poecilia reticulata. Pesticide Biochem. Physiol. 101: 132-141.

Shahzad M, Javed MT, Shabir S, Irfan M and Hussain R, 2012. Effects of feeding urea and copper sulphate in different combinations on live body weight, carcass weight, percent weight to body weight of different organs and histopathological tissue changes in broilers. Exp. Toxicol. Pathol. 64:141- 147

Sun X, Li J, Zhao H, Wang Y, Liu J, Shao Y, Xue Y and Xing M, 2018. Synergistic effect of copper and arsenic upon oxidative stress, inflammation and autophagy alterations in brain tissues of Gallus gallus. J. Inorg. Biochem. 178:54-62.

Sunaina A and Ansari BA, 2016. Oxidative stress biomarkers in assessing arsenic tri oxide toxicity in the Zebrafish, Danio rerio. Int. J. Fish. Aquat. Syst. 4:8-13.

Taweel A, Othman MS and Ahmad A, 2011. Heavy metals concentration in different organs of tilapia fish (Oreochromis niloticus) from selected areas of Bangi, Selangor, Malaysia. Afr. J. Biotech. 10:11562-11566. .

Tripathi G and Shasmal J, 2011. Concentration related responses of chlorpyriphos in antioxidant, anaerobic and protein synthesizing machinery of the freshwater fish, Heteropneustes fossilis. Pesticide Biochem. Physiol. 99:215-220.

Tripathi V and Yadav R, 2015. Effect of Pesticide (Organophosphorus) on aquatic fish Labeo Rohita. Int. J. Chem. Sci., 13:625-640.

Varsha G, Malik DS and Dinesh K, 2017. Risk assessment of heavy metal pollution in middle stretch of river Ganga: an introspection. Int. Res. J. Environ. Sci. 6: 62-71.

\section{Contribution of Authors}

Rehman T: Planned and conducted the experiment Naz S: Planned and conducted the experiment Hussain R: Analyzed the data and wrote the manuscript

Chatha AMM: Involved in interpretation of results and manuscript preparation.

Ahmad F: Involved in interpretation of results and manuscript preparation.

Yamin A: Planned and conducted the experiment Akram R: Involved in interpretation of results and manuscript preparation.

Naz H: Involved in interpretation of results and manuscript preparation.

Shaheen A: Planned and conducted the experiment 